\title{
Violaxanthin and diadinoxanthin de-epoxidation in various model lipid systems*
}

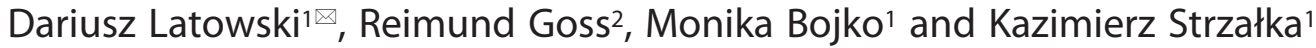 \\ 1Department of Plant Physiology and Biochemistry, Jagiellonian University, Kraków, Poland; ${ }^{2}$ nnstitute of Biology I, Plant Physiology, University of \\ Leipzig, Leipzig, Germany
}

The xanthophyll cycle is an important photoprotective process functioning in plants. One of its forms, the violaxanthin $(V x)$ cycle, involves interconversion between: $\mathrm{Vx}$, antheraxanthin $(\mathrm{Ax})$ and zeaxanthin (Zx). Another kind of the xanthophyll cycle is the diadinoxanthin (Ddx) cycle in which interconversion between $\mathrm{Ddx}$ and diatoxanthin (Dtx) occurs. In this study an information on molecular mechanism and regulation of these two types of the xanthophyll cycle is presented. The influence of lipids on the de-epoxidation of the xanthophyll cycle pigments was investigated, with special focus put on the significance of physical properties of the aggregates formed by inverted lipid micelles, which are necessary for activity of the xanthophyll cycle enzymes. In particular, thickness of the hydrophobic fraction of the aggregates, size of the inverted micelles, suggested by mathematical description of the structures and solubility of Vx and Ddx in various kind of lipids were studied. Obtained results show that the rate of de-epoxidation is strongly dependent on the physicochemical properties of the lipids used. The key role for enzyme activation play non-bilayer lipids and the parameters of inverted micelles such as thickness, fluidity of hydrophobic core and their diameter. The presented results show that MGDG and other non-lamellar lipids like different forms of phosphatidylethanolamine are necessary for the $\mathrm{Vx}$ and Ddx de-epoxidation because they provide the threedimensional structures, which are needed for the binding of de-epoxidases and for the accessibility of $V x$ and Ddx to these enzymes.

Key words: violaxanthin cycle, diadinoxanthin cycle, de-epoxidation, lipids

Received: 17 October, 2011; accepted: 01 March, 2012; available on-line: 17 March, 2012

\section{INTRODUCTION}

Violaxanthin and diadinoxanthin de-epoxidation are photo-protective reactions enagaged in process called xanthophyll cycle. There are few variants of the xanthophyll cycle (Latowski et al., 2011). In the most common variant of this process, known also as the violaxanthin cycle, under strong light conditions, enzyme violaxanthin de-epoxidase (VDE) catalyses de-epoxidation of violaxanthin $(\mathrm{Vx})$ to zeaxanthin $(\mathrm{Zx})$ via antheraxanthin $(\mathrm{Ax})$ as an intermediate product. This type of the cycle exists in higher plants, ferns, mosses and some group of algae (Stransky \& Hager, 1970; Adamska, 1997). Another deepoxidase, called diadinoxanthin de-epoxidase (DDE) is one of the enzymes of another kind of xanthophyll cycle known as diadinoxanthin cycle. This cycle functions in several algal groups (diatoms, phaeophytes, dinophytes and haptophytes). The most known representatives of these groups are Phaeodactylum tricornutum and Cyclotella meneghiniana. DDE catalyses de-epoxidation of monoepoxide diadinoxanthin (Ddx) to diathoxanthin (Dtx) (Stransky \& Hager, 1970). Although DDE has substantially higher $\mathrm{pH}$ optimum than VDE it is known that both types of the xanthophyll cycle are similar on molecular level (Grouneva et al., 2006). In both of them the reducing agent needed for de-epoxidation is ascorbate (Asc) (Hager, 1969, Yamamoto 1979, Grouneva et al., 2006) and additionally, as we showed previously, reverse hexagonal phase forming lipids (monogalactosyldiacylglycerol - MGDG, phosphatidylethanolamine - PE) are indispensible for this reaction (Latowski et al., 2002, 2004, Goss et al., 2005, 2007).

Both VDE and DDE are water soluble lumenal proteins that undergo a conformational change when $\mathrm{pH}$ drops due to formation of the light-driven proton gradient across the thylakoid membrane (Pfündel \& Bilger, 1994, Jakob et al., 2001, Hager \& Holocher, 1994). The change in enzyme conformation is accompanied by the functional binding of the enzymes to the thylakoid membrane, where the substrates: Vx or Ddx are located.

\section{MATERIALS AND METHODS}

VDE was isolated from 7-day old wheat plants (Triticum aestivum) (Hager \& Holocher, 1994; Goss et al., 2005) and Vx was extracted from daffodil (Narcissus poeticus) petals (Frommolt et al., 2001). DDE and Ddx were prepared from thylakoid membranes of C. meneghiniana (strain 1020-1a). C. meneghiniana was obtained from the Sammlung von Algenkulturen Goettingen (SAG) and was grown as a batch culture in silica enriched ASP (Provasoli et al., 1957; Lohr \& Wilhelm, 2001). Reaction assay contained $0.33 \mu \mathrm{M} V \mathrm{x}$ or Ddx, different concentration of MGDG or one of the different types of PE varying in the acyl residues and $5 \%$ ethanol $(\mathrm{v} / \mathrm{v})$ in 40 $\mathrm{mM}$ MES, pH 5.2, with $10 \mathrm{mM} \mathrm{KCl}$ and $5 \mathrm{mM} \mathrm{MgCl}_{2}$. Reaction was started by Asc addition (final conc. 30

e-mail: dariuszlatowski@gmail.com

*Presented at the 16th International Symposium on Carotenoids, 17-22 July, 2011, Kraków, Poland

Abbreviations: Asc, ascorbate; Ax, antheraxanthin; DDE, diadinoxanthin de-epoxidase; Ddx, diadinoxanthin; DGDG, digalactosyldiacylglycerol; Dtx, diatoxanthin; DPH, diphenylhexatriene; MGDG, monogalactosyldiacylglycerol; PC, phosphatidylcholine; $\mathrm{PE}$, phosphatidylethanolamine; PE1, 1,2-dioleoyl-sn-glycero-3-phosphatidylethanolamine; PE2, 1,2-dilinoleoyl-sn-glycero-3-phosphatidylethanolamine; PE3, 1,2-dilinoleinoyl-sn-glycero-3-phosphatidylethanolamine; PE-C16, 1,2-dipalmitoyloleoyl-sn-glycero-3-phosphatidylethanolamine; VDE, violaxanthin de-epoxidase; Vx, violaxanthin; ZE, zeaxanthin epoxidase; Zx, zeaxanthin. 
$\mathrm{mM}$ ) and samples were collected at 0,2,5 and $10 \mathrm{~min}$. Reaction was stopped by mixing $700 \mathrm{ml}$ of assay mixture with $50 \mathrm{ml}$ of $1 \mathrm{M} \mathrm{KOH}$, then centrifuged. Pellets, containing pigments, were analyzed by reverse phase HPLC column (Nucleosil C-18) as describe by Latowski et al. (2002). Fluidity of hydrophobic fraction of aggregates of inverted micelles composed of one of the following lipids: MGDG, PE from eggs, 1,2-dioleoyl-sn-glycero-3 phosphatidylethanolamine (PE1) or 1,2-dilinoleoyl-snglycero-3-phosphatidylethanolamine (PE2) or 1,2-dilinoleinoyl-sn-glycero-3-phosphatidylethanolamine (PE3) was assessed by by measuring steady-state fluorescence polarization anisotropy of diphenylhexatriene (DPH) with lipid:DPH ratio of 500. Final lipid concentration was $0.5 \mathrm{mM}$, emission and excitation wavelengths 425 $\mathrm{nm}$ and $355 \mathrm{~nm}$ respectively.

\section{RESULTS AND DISCUSSION}

Obtained results show that the activity both of VDE and DDE are strongly dependent not only on the presence of non-lamellar lipids (Latowski et al., 2004; Goss et al., 2005; Goss et al., 2007) but also on physicochemical parameters of inverted micelles created by these lipids. These parameters are: fluidity of hydrophobic core (Fig. 1), its thickness (Fig. 2) and diameter of micelles created by the tested lipids (Fig. 3 and 4).

The dependence between the conversion of $\mathrm{Vx}$ into $\mathrm{Ax}$ and Zx as well as Ddx to Dtx and fluidity of the hydrophobic fraction of inverted micelles, showed that the highest activity was observed in the case of PE1, PE2 and MGDG for VDE and DDE, respectively, although fluidity of structures formed by these lipids was relatively low (Fig. 1).

Studies on the effect of thickness of the hydrophobic core of the aggregates on VDE and DDE activity revealed that DDE activity was about $25 \%$ lower in PEC16 comparing PE1 micelles while activity of VDE in PE-C16 micelles was lower by 50\% comparing the micelles composed of PE1. PE1 and PE-C16 are esterified with fatty acids having one double bond but differing in their length. PE1 is about $6 \AA$ longer than PE-C16.

These results suggest that both VDE and DDE need special value of membrane fluidity and thickness of the hydrophobic core of the tested lipid structures that provides optimum conditions for the de-epoxidation. When the fluidity of lipids is too high, their arrangement becomes disordered and this enables more possible orientations the epoxy-xanthophylls, resulting in a decrease in number of molecules having orientation ensuring accessibility by de-epoxidising enzymes.

Similarly, the membrane thickness influences the position of pigment molecules. When the thickness of the membrane is lower than the length of the xanthophyll molecule, the angle of pigment molecules in relation to the membrane plane changes to guarantee of beta ionon rings' interaction with polar head of lipids. The change of pigments orientation in the membrane is likely preventing efficient penetration of these molecules into active site of the enzyme.

Another parameter studied was the size of the inverted micelles. Diameter of the micelles was about 7 to 9 $\mathrm{nm}$, when they were created by MGDG only, and about $15 \mathrm{~nm}$ and 20 to $21 \mathrm{~nm}$ when mixtures of MGDG and DGDG (50:50 and 85:15, respectively) were used (Sprague \& Staehelin, 1984). As Figs. 3 and 4 show, the larger the diameter of the micelles the smaller rate of de-epoxidation both for VDE and DDE. These results

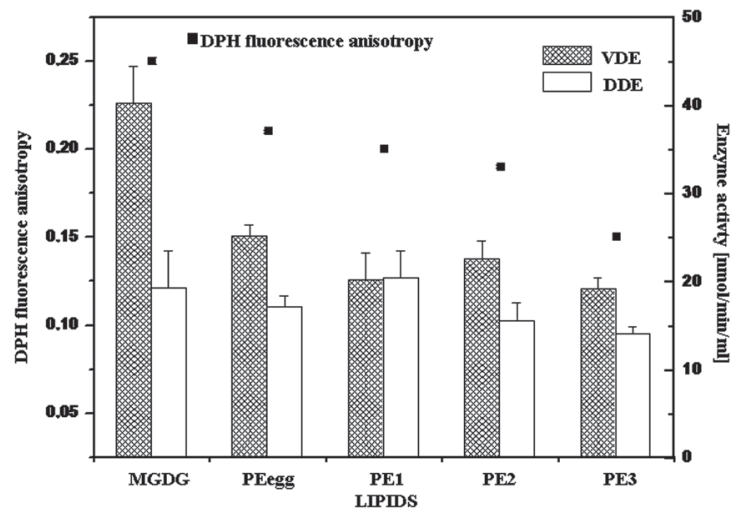

Figure 1. Effect of fluidity of hydrophobic fraction of inverted micelles composed of different types of lipids on VDE and DDE activity.

Fluidity was estimated by measuring DPH fluorescence anisotropy. DDE, diadinoxanthin de-epoxidase; DPH, diphenylhexatriene; MGDG, monogalactosyldiacylglycerol; PE1, 1,2-dioleoyl-sn-glycero-3-phosphatidylethanolamine; PE2, 1,2-dilinoleoyl-sn-glycero3-phosphatidylethanolamine; PE3, 1,2-dilinoleinoyl-sn-glycero3-phosphatidylethanolamine; VDE, violaxanthin de-epoxidase.

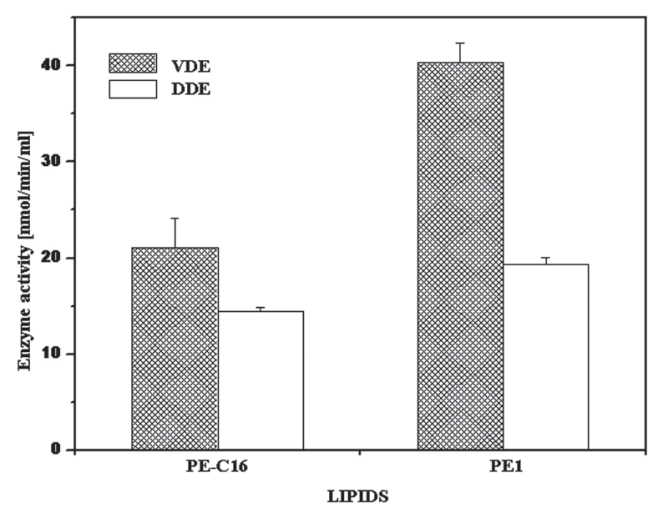

Figure 2. Effect of the thickness of the hydrophobic fraction of inverted hexagonal structures on VDE and DDE activity.

Hydrophobic fraction of the inverted hexagonal structures composed by PE1 is about $1.2 \mathrm{~nm}$ broader than hydrophobic structures created by PE-C16. DDE, diadinoxanthin de-epoxidase; PE1, 1,2-dioleoyl-sn-glycero-3-phosphatidylethanolamine; PE-C16, 1,2-dipalmitoyloleoyl-sn-glycero-3-phosphatidylethanolamine; VDE, violaxanthin de-epoxidase.

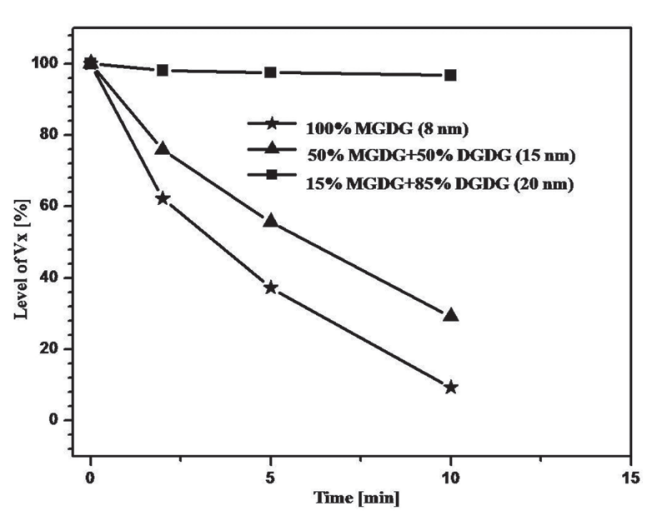

Figure 3. Effect of diameter of inverted hexagonal micelles on violaxanthin de-epoxidation.

DGDG, digalactosyldiacylglycerol; MGDG, monogalactosyldiacylglycerol. 


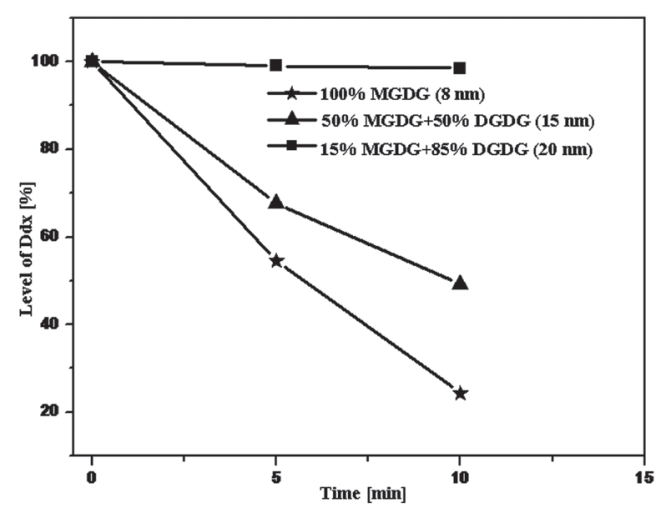

Figure 4. Effect of diameter of inverted hexagonal micelles on diadinoxanthin de-epoxidation.

DGDG, digalactosyldiacylglycerol; MGDG, monogalactosyldiacylglycerol.

may be explained assuming the larger the diameter of the micelles the longer is the way the epoxy-xanthophyll molecules have to diffuse to reach the de-epoxidising enzymes bound to the lipid micelles, hence smaller rate of de-epoxidation.

Obtained results suggest that mutual orientation of enzymes and substrates as well as solubilization and dilution of pigments by lipids are the main factors controlling molecular mechanism of de-epoxidation both $\mathrm{Vx}$ and Ddx.

\section{REFERENCES}

Adamska I (1997) ELIPs-light-induced stress proteins. Review. Physiol Plant 100: 794-805.

Frommolt R, Goss R, Wilhelm C (2001) The de-epoxidase and epoxidase reactions of Mantoniella squamata (Prasinophyceae) exhibit different substrate-specific reaction kinetics compared to spinach. Planta 213: 446-456.

Goss R, Latowski D, Grzyb J, Vieler A, Lohr M, Wilhelm C, Strzałka K (2007) Lipid dependence of diadinoxanthin solubilization and de-epoxidation in artificial membrane systems resembling the lipid composition of the natural thylakoid membrane. Biochim Biophys Acta 1768: $67-75$

Goss R, Lohr M, Latowski D, Grzyb J, Vieler A, Wilhelm C, Strzałka $\mathrm{K}$ (2005) Role of hexagonal structure forming lipids in diadinoxanthin and violaxanthin solubilization and de-epoxidation. Biochemistry 44: 4028-4036.

Grouneva I, Jakob T, Wilhelm C, Goss R (2006) Influence of ascorbate and $\mathrm{pH}$ on the activity of the diatom xanthophyll cycle-enzyme diadinoxanthin de-epoxidase. Physiol Plant 126: 205-211.

Hager A (1969) Lichtbedingte pH-Erniedrigung in Einem Chloroplastenkompartiment als Ursache der Enzymatischen ViolaxanthinZeaxanthin-Umwandlung; Beziehungen zur Photophosphorylierung. Planta 89: 224-243.

Hager A, Holocher K (1994) Localization of the xanthophyll-cycle enzyme violaxanthin de-epoxidase within the thylakoid lumen and abolition of its mobility by a (light-dependent) $\mathrm{pH}$ decrease. Planta 192: 581-589.

Jakob T, Goss R, Wilhelm C (2001) Unusual pH-dependence of diadinoxanthin de-epoxidase activation causes chlororespiratory induced accumulation of diatoxanthin in the diatom Phaeodactylum tricornutum. J Plant Physiol 158: 383-390.

Latowski D, Akerlund HE, Strzałka K (2004) Violaxanthin de-epoxidase, the xanthophyll cycle enzyme, requires lipid hexagonal structures for its activity. Biochemistry 43: 4417-4420.

Latowski D, Kruk J, Burda K, Skrzynecka-Jaskier M, Kostecka-Gugała A, Strzałka K (2002) Kinetics of violaxanthin de-epoxidation by violaxanthin de-epoxidase, a xanthophyll cycle enzyme, is regulated by membrane fluidity in model lipid bilayers. Eur J Biochem 269: 4656-4665.

Latowski D, Kuczyńska P, Strzałka K (2011) Xanthophyll cycle — a mechanism protecting plants against oxidative stress. Redox Rep 16: 78-90.

Lohr M, Wilhelm C (2001) Xanthophyll synthesis in diatoms: quantification of putative intermediates and comparison of pigment conversion kinetics with rate constants derived from a model. Planta 212: 382-391.

Pfündel EE, Bilger W (1994) Regulation and possible function of the violaxanthin cycle. Photosynth Res 42: 89-109.

Provasoli L, McLaughlin JJA, Droop MR (1957) The development of artificial media for marine algae. Arch Mikrobiol 25: 392-428.

Sprague GS, Staehelin LA (1984) Effect of reconstitution method on the structural organization of isolated chloroplast membrane lipids. Biochim Biophys Acta 777: 306-322.

Stransky H, Hager A (1970) The carotenoid pattern and the occurrence of the light-induced xanthophyll cycle in various classes of algae. VI. Chemosystematic study. Arch Mikrobiol 73: 315-323.

Yamamoto HY (1979) Biochemistry of the violaxanthin cycle in higher-plants. Pure Appl Chem 51: 639-648. 